PROFESIONALES Y HERRAMIENTAS PARA EL DESARROLLO LOCAL Y SUS SINERGIAS TERRITORIALES. EVALUACIÓN Y PROPUESTAS DE FUTURO IX Coloquio Nacional de Desarrollo Local del GTDL-AGE 

ANTONIO MARTÍNEZ PUCHE, XAVIER AMAT MONTESINOS, ISABEL SANCHO CARBONELL y DANIEL SANCHIZ CASTAÑO (EDS.)

\section{PROFESIONALES Y HERRAMIENTAS PARA EL DESARROLLO LOCAL Y SUS SINERGIAS TERRITORIALES. EVALUACIÓN Y PROPUESTAS DE FUTURO}

IX Coloquio Nacional de Desarrollo Local del GTDL-AGE

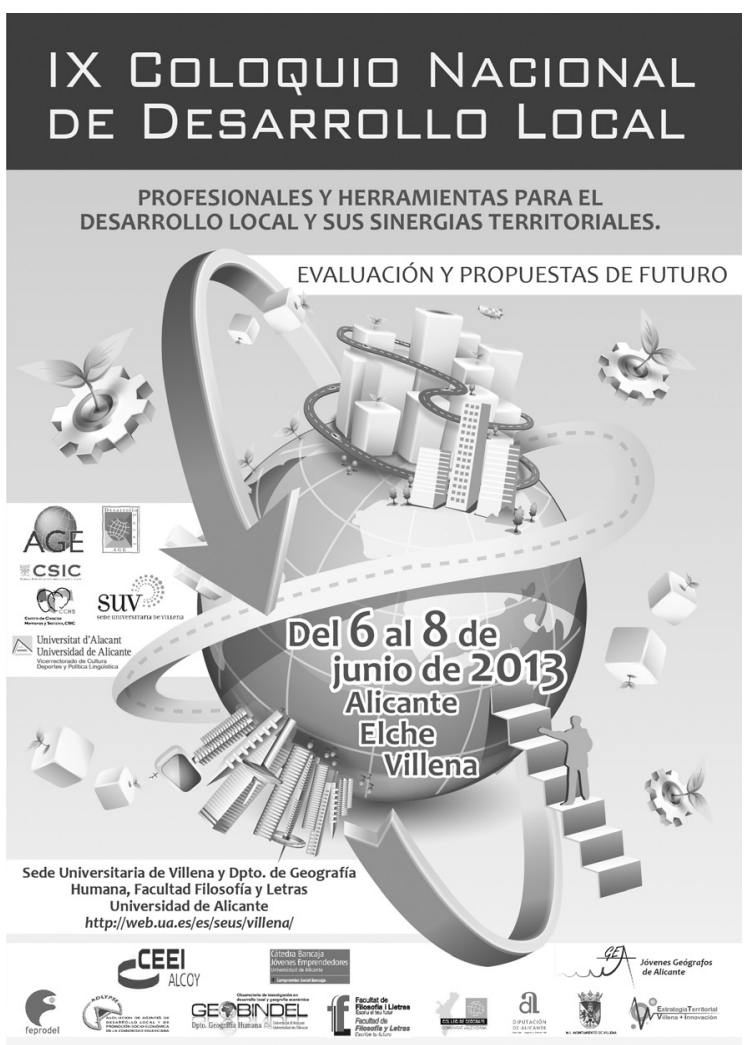


Este libro ha sido examinado y valorado por evaluadores ajenos a la Universidad de Alicante, con el fin de garantizar la calidad científica del mismo.

Publicacions de la Universitat d'Alacant

03690 Sant Vicent del Raspeig

Publicaciones@ua.es

http://publicaciones.ua.es

Telèfon: 965903480

(C) Antonio Martínez Puche, Xavier Amat Montesinos,

Isabel Sancho Carbonell y Daniel Sanchiz Castaño (eds.), 2016

(C) d'aquesta edició: Universitat d'Alacant

ISBN: 978-84-16724-00-0

Dipòsit legal: A 92-2016

Disseny de coberta: candela ink

Composició: Página Maestra (Miguel Ángel Sánchez Hernández)

Impressió i enquadernació: Guada Impresores

\section{unte \\ Unión de Editoriales
Universitarias Españolas \\ WWW.une.es
WWA}

Esta editorial es miembro de la UNE, cosa que garantiza la difusión y comercialización nacional y internacional de sus publicaciones.

Reservados todos los derechos. Cualquier forma de reproducción, distribución, comunicación pública o transformación de esta obra sólo puede ser realizada con la autorización de sus titulares, salvo excepción prevista por la ley. Diríjase a CEDRO (Centro Español de Derechos Repográficos, www.cedro.org) si necesita fotocopias o escanear algún fragmento de esta obra. 


\title{
MONTAÑA MEDITERRÁNEA Y ESPACIOS PROTEGIDOS EN ESPAÑA: OPORTUNIDADES Y AMENAZAS PARA SU DESARROLLO LOCAL
}

\author{
$M^{a}$ Luisa Gómez Moreno \\ Departamento de Geografía de la Universidad de Málaga. \\ geolugom@uma.es
}

RESUMEN

La comunicación analiza, desde la epistemología del desarrollo local, los factores que impiden que las áreas de montaña mediterránea se beneficien de las oportunidades derivadas de su declaración como espacios naturales protegidos. Así mismo, describe las oportunidades de mejora implícitas en la Estrategia Euroepa 2020 y en la Red Natura 2000 para superar estos obstáculos. En función de éstas, se proponen nuevas formas de gestión aplicables tanto a las áreas de montaña ricas en biodiversidad y, por tanto, susceptibles de ser protegidas, como a aquellas objeto de regulaciones menos restrictivas pero también con frágiles recursos territoriales.

Palabras clave: Desarrollo local y rural; gestión de espacios naturales protegidos; áreas de montaña mediterránea.

\section{MEDITERRANEAN MOUNTAIN AREAS AND PROTECTED SPACES \\ IN SPAIN: OPPORTUNITIES AND TREATS FOR ITS LOCAL DEVELOPMENT}

\section{Abstract}

This paper analyses, from the standpoint of local development, the factors impeding Mediterranean mountain areas from benefiting from the opportunities offered by their declaration as protected natural areas. In addition, it describes opportunities for improvement, taking into account the Europe 2020 Strategy and the Natura 2000 network. To overcome these shortcomings, we propose new forms of management applicable to mountain areas, both those containing 
specific biodiversity assets, and thus eligible for protection, and those subject to less restrictive regulations but nevertheless featuring fragile territorial resources.

Key words: Management of protected areas; Mediterranean mountains; rural and local development.

\section{INTRODUCCIÓN. MARCO TEÓRICO Y METODOLOGÍA}

La localización predominante de los parques naturales españoles en las zonas de montaña mediterránea se deriva de la evolución de las funciones que estas áreas han desempeñado en los sistemas territoriales del capitalismo industrial y del postindustrial. Como consecuencia de esta evolución, aunque este hecho lleva aparejado una mayor visibilidad y, por consiguiente, una mayor competitividad en la oferta de espacios recreativos con altos valores ambientales demandados por las sociedades urbanas postindustriales, varios factores dificultan que esta oportunidad sea una herramienta real para superar los problemas de esta categoría de zonas.

Los objetivos de este trabajo ${ }^{1}$ son:

- Exponer estos factores desde el marco teórico del desarrollo local.

- Aplicar este análisis al caso de estudio del parque natural de Sierra de las Nieves (Málaga, España);

- Proponer nuevas formas de gestión para superar estos obstáculos basándose en la Estrategia Europea 2020 y en la ampliación de la superficie protegida en áreas de montaña derivada de la aplicación tanto de la Red Natura 2000 como de la Ley 42/2007 (de 13 de diciembre) de Patrimonio Natural y Biodiversidad.

Para el desarrollo de estos objetivos se han aplicado de forma interrelacionada dos marcos teóricos: el del desarrollo local, en los términos de capital territorial DEMATTEIS y GOVERNA, 2005) y sus componentes, analizando la modificación de éstos en función de la evolución desde la economía orgánica (WRIGLEY, 1988) hacia el capitalismo industrial y el capitalismo postindustrial (GÓMEZ, 2008).

En el contexto de la economía orgánica, la función de la montaña mediterránea era la producción de alimentos y materias primas basándose en la

1 Esta comunicación forma parte de los resultados de la suma de dos proyectos: el Proyecto I+D+I, del Ministerio de Ciencia e innovación del Gobierno de España (referencia SEJ2007-67690), con Carmen Ocaña Ocaña como investigadora responsable, y el Proyecto de Excelencia de la Consejería de Innovación, ciencia y empresa de la Junta de Andalucía (Referencia P07_HUM_03049), con Ma Luisa Gómez Moreno, como investigadora responsable. Parte sustantiva del mismo se llevó como póster al 5th International Conference Rural Space and Local Development. Rural Mountain Areas. Esperience, preservation, trends and future challenges", Sighetu (Rumanía) 18 a 22 de julio de 2012, siendo posteriormente publicado en Journal of Settlements and Spatial Planning. 
interacción entre ager y saltus bajo la misma unidad territorial (el concejo) cuya estructura de gobierno redactaba y aplicaba las normas de gestión. En esta etapa, agentes y territorios estaban, por tanto, vinculados a través de la gestión.

El capitalismo industrial supone la pérdida de competitividad de estos espacios con dos consecuencias fundamentales: el éxodo rural (pérdida de la componente capital humano en términos cualitativos y cuantitativos) y la segregación de la interacción de ager y saltus, ya que el primero entra en proceso de abandono desigual por la falta de rentabilidad de sus producciones y el segundo es, frecuentemente, objeto de políticas forestales llevadas a cabo por nuevos agentes desligados del territorio y su trayectoria y que aplican sistemas de gestión basados en los conocimientos científico-académicos (conocimientos reglados) y no en el conocimiento concreto de la componente biótica y abiótica del capital territorial (componente denominada capital cognitivo local). En este sentido, hay que destacar que la finalidad de estas políticas forestales se centraba, en no pocas ocasiones, en el beneficio de áreas externas a la montaña, como es el caso de la prevención de los efectos de las inundaciones en las ciudades situadas en las llanuras aluviales o el entarquinamiento de los pantanos que entonces se construyen.

Finalmente, el capitalismo postindustrial lleva aparejada una compleja y contradictoria sucesión de funciones. Por orden cronológico, el paradigma de la conservación del que podemos considerar ejemplo más significativo las propuestas de la UICN en su formulación de 1978 (IUCN, 1994). Pero casi simultáneamente tiene lugar la valoración por la sociedad urbana de los denominados "Rural Goods and Services (RGS)" (ESPARCIA y BUCIEGA, 2005) que pasan a convertirse en objeto de demanda bajo formas turísticas y recreativas y que las zonas de montaña pueden proporcionar por la menor presencia de los procedimientos tecnológicos en sus procesos productivos, que ya hemos señalado y que es la clave de esa mayor competitividad también enunciada en la introducción. Podríamos considerar este proceso como uno de los factores de la plurifuncionalidad de los espacios rurales. Finalmente, a partir de 1992, la formulación del paradigma de la sostenibilidad que, teóricamente, proporciona la base para la compatibilidad de uso y conservación de zonas con altos valores ambientales.

La combinación de estos tres paradigmas en términos de la teoría del desarrollo local aplicado a las zonas de montaña españolas puede expresarse en los siguientes términos. En primer lugar, la difusión de la localización de los parques naturales en las áreas de montaña a partir de la Ley 4/1989 en su aplicación por los gobiernos autonómicos. La aplicación de esta ley en términos de gestión ha supuesto la continuidad de los técnicos forestales como agentes y la asunción de los planteamientos de las citadas propuestas de la UICN de 1978, esto es, la potenciación de la conservación y de la educación ambiental como objetivos fundamentales en detrimento de las comunidades autóctonas, cuyo 
desarrollo es, teóricamente, también uno de los objetivos de la gestión. En este sentido, la demarcación de los parques naturales viene a subrayar la separación ager saltus que ya introdujo la gestión forestal, conduciendo al típico mapa de "islas de protección". A su vez, este objetivo de la educación ambiental ha convergido con la demanda de RGS por las sociedades urbanas alóctonas, de modo que los agentes que priman en la gestión del territorio no forman parte del capital humano del territorio afectado por la protección.

Siguiendo siempre con el caso de España, la asimilación del paradigma de la sostenibilidad no se ha traducido en un reforzamiento práctico del papel de las comunidades en la gestión de los espacios naturales protegidos, sino que se ha aplicado el siguiente razonamiento: reforzando la función recreativa de los parques naturales a través de los Planes de Uso Público, se incrementará el número de visitantes a éstos que redundará, de forma indirecta, en la demanda de servicios privados proporcionados por el tejido productivo local: hoteles, restaurantes, empresas de ocio (Pulido, 2008). Sin embargo, no parece ser un problema exclusivo de España como indican los planteamientos de Benos, Cazenave y Milian (BENOS, CAZENAVE, y MILIAN, 2007: 47) "consiste en interrogarnos sobre lo rural mismo a través de la protección de la naturaleza, confiriéndole un valor normativo: ¿qué aporta la protección a la comprensión de lo rural en tanto que categoría de razonamiento y de selección geográfica? ¿cómo interactúa la protección con los otros componentes estructurales? ¿en qué modifica la protección los otros componentes estructurales?"

Estos planteamientos fueron tenidos en cuenta para enunciar parte de los objetivos y la metodología del proyecto cuyos resultados se exponen en este artículo (ver nota 1 y OCAÑA et al en prensa). Este proyecto se centró en la experimentación de metodologías para la medición de la aptitud y la capacidad de carga para usos recreativos, aplicables a la ordenación y gestión de los recursos de los espacios naturales protegidos. Asumiendo una perspectiva amplia de los conceptos de capacidad de carga y de acogida, en la línea de conciliar actividad económica y protección (que ratificarían después los planteamientos de la Estrategia Europa 2020) incorporó como parte esencial de la evaluación las posibles implicaciones de la prácticas recreativa en el desarrollo de las localidades vinculadas a este tipo de espacios, con la intención de constatar las implicaciones reales existentes entre sus modelos de gestión, la promoción de las actividades turístico-recreativas y el desarrollo local (PULIDO, 2008).

Centrándonos en este último objetivo, para su desarrollo se recurrió a tres tipos de procedimientos: el análisis de la normativa de gestión; el comportamiento de los senderistas en relación con el tejido productivo rural y el análisis del tejido productivo rural con especial atención a los actores y sectores relacionados con las actividades recreativas. De estos procedimientos, en este artículo vamos a exponer los resultados correspondientes a los dos primeros. El ámbito de estudio fue el parque natural de la Sierra de las Nieves (Andalucía, España) 
fue seleccionado como área de estudio por diferentes razones, entre las que se cuenta ser significativo de esta nueva función de las áreas de montaña a la que aludíamos. En él se llevó a cabo una encuesta (con un total de 350 respuestas entre octubre de 2010 y junio de 2011), cuyo cuestionario comprendía 3 secciones en relación con los objetivos globales del proyecto características del visitante (origen, edad, sexo, profesión); motivación y percepción de la saturación, fundamental para estimar la capacidad de carga social, y satisfacción con el recorrido realizado; y, un último bloque, que es el abordado en este epígrafe, que respondió al marco teórico del desarrollo rural sostenible, destinándose a conocer tanto la composición y alcance del gasto realizado por el usuario de los senderos en el tejido productivo de los municipios del entorno como su grado de satisfacción con los servicios utilizados, incluyendo en este caso tantos los públicos (equipamientos del parque) como los privados.

Finalmente, para el análisis de estas fuentes se ha tenido en cuenta la bibliografía y el estudio del contexto nacional y regional de la gestión de los parques naturales y su incidencia en el desarrollo rural.

\section{RESULTADOS Y DISCUSIÓN}

2.1. ¿Por qué los parques naturales no están contribuyendo a la dinamización socioeconómica de la montaña mediterránea en Andalucía?

Para dar respuesta a dos de los planteamientos de BENÓS, CAZENAVE y MILLIAN (2007), ¿cómo interactúa la protección con los otros componentes estructurales? ¿en qué modifica la protección los otros componentes estructurales?, hemos recurrido al análisis de fuentes bibliográficas. 23 años después de que se promulgara en Andalucía el Inventario de Espacios Naturales Protegidos de Andalucía, que materializó la aplicación de la Ley 4/1989 de Conservación de los Espacios Naturales y de la Flora y Fauna Silvestres, la observación en el ZOIDO y CARAVACA (2005) del "tipo de desarrollo territorial" en Andalucía (ver cuadro 1) revela que las áreas de montaña mediterránea en las que se ubican parques naturales no se diferencian de las zonas de montaña que carecen de éstos por un mayor dinamismo económico. Por lo tanto, esta figura de conservación no está interactuando de forma positiva con los restantes componentes del tejido socioeconómico rural. ¿Por qué? En nuestra opinión, estas son las causas que explican esta carencia de sinergia:

- Sus bases demográficas. Como consecuencia del intenso éxodo ligado a la fase del capitalismo industrial, el capital humano está envejecido y en decrecimiento cuantitativo. Ello supone despoblación y una débil y pasiva estructura empresarial incapaz de identificar las nuevas actividades económicas ligadas a la presencia del parque natural.

- La protección no se articula con las bases del sistema productivo. Así, no mejora ni compensa la carencia de rentabilidad del ager y la gestión 
productiva del saltus incide débilmente en las rentas de los habitantes. Estos hechos implican el abandono de los usos que constituían las bases del sistema agroambiental que, precisamente, había justificado su reconocimiento como area protegida en no pocos casos.

- La histéresis de los principios de gestión iniciales de la UICN, inadecuados para las estructuras sociales y territoriales de las montañas mediterráneas europeas. Pero si bien la UICN ha reconocido la necesidad

Cuadro 1. Superficie total, número de visitantes e índice de desarrollo territorial de los parques naturales andaluces

\begin{tabular}{|l|c|c|c|c|c|}
\hline \multicolumn{1}{|c|}{$\mathbf{1}$} & \multicolumn{2}{c|}{$\mathbf{2}$} & \multicolumn{2}{c|}{$\mathbf{3}$} & $\mathbf{4}$ \\
\hline \multicolumn{1}{|c|}{ Parque natural } & $\begin{array}{c}\text { Superficie } \\
\text { ha }\end{array}$ & $\begin{array}{c}\text { \% } \\
\text { sup. }\end{array}$ & $\begin{array}{c}\text { Código } \\
\text { nisitan- } \\
\text { tes 2009 }\end{array}$ & $\begin{array}{c}\text { \% } \\
\text { visit. }\end{array}$ & $\begin{array}{c}\text { desarrollo } \\
\text { territorial }\end{array}$ \\
\hline Natural Sierra María-Los Vélez & 22562 & 1,4 & 15938 & 1,1 & \\
\hline Sierra Nevada & 88966 & 5,7 & & 0,0 & 1 \\
\hline Los Alcornocales & 167767 & 10,7 & 11335 & 0,8 & 3 \\
\hline Sierra de Grazalema & 53411 & 3,4 & 101521 & 7,3 & 3 \\
\hline Sierra de Cardeña y Montoro & 38449 & 2,5 & 11799 & 0,8 & 2 \\
\hline Sierra de Hornachuelos & 60032 & 3,8 & 14482 & 1,0 & 3 \\
\hline Sierras Subbéticas & 32056 & 2,1 & 26877 & 1,9 & 2 \\
\hline Sierra Nevada & 85883 & 5,5 & 83003 & 5,9 & 1 \\
\hline Sierra de Baza & 53649 & 3,4 & 1454 & 0,1 & 1 \\
\hline Sierra de Castril & 12696 & 0,8 & 8557 & 0,6 & 1 \\
\hline Sierra de Huétor & 12128 & 0,8 & 7964 & 0,6 & \\
\hline Sierras de Tejeda, Almijara y Alhama & 40663 & 2,6 & 3587 & 0,3 & 1 \\
\hline Sierra de Aracena y Picos de Aroche & 186827 & 12,0 & 8380 & 0,6 & 1 \\
\hline Despeñaperros & 7649 & 0,5 & 10504 & 0,8 & \\
\hline Sierra de Andújar & 74774 & 4,8 & 9471 & 0,7 & 3 \\
\hline Sierra Mágina & 19961 & 1,3 & 25499 & 1,8 & 2 \\
\hline Sierras de Cazorla, Segura y Las Villas & 209920 & 13,4 & 197282 & 14,1 & 1 \\
\hline Montes de Málaga & 4996 & 0,3 & 12716 & 0,9 & \\
\hline Sierra de las Nieves & 20163 & 1,3 & 57000 & 4,1 & 3 \\
\hline Sierra Norte de Sevilla & 177484 & 11,4 & 23505 & 1,7 & 1 \\
\hline Total & 1562165 & 100,0 & 1395391 & 100,0 & 1 \\
\hline
\end{tabular}

Fuente: elaboración propia. 
de fortalecer la participación de los habitantes locales en la gestión de la categoría V (DUDLEY, 2008), la nueva ley (Ley 42/2007 de 13 de diciembre del patrimonio natural y la biodiversidad) mantiene los fundamentos originales cuya aplicación está suponiendo el debilitamiento de la capacidad de los habitantes como agentes en la gestión del parque frente al fortalecimiento del rol de los visitantes a través de los objetivos que vinculan la conservación de la biodiversidad con la educación ambiental. En otras palabras, esta función de la montaña mediterránea como localización predominante de los parques naturales potencia la conservación de la biodiversidad y la oferta de áreas recreativas al aire libre en detrimento de la mejora de la integración de sus habitantes en el tejido soicoproductivo europeo.

\subsection{Caso de estudio: Parque natural de Sierra de las Nieves, Málaga, España}

La delimitación y gestión del parque natural Sierra de las Nieves responde al desarrollo que la Junta de Andalucía (organismo de gobierno regional) hace de la Ley 4/1989 de 27 de marzo Conservación de los Espacios Naturales y de la Flora y Fauna Silvestres que, al producirse ya bajo el traspaso de competencias, va a aplicarse en el territorio andaluz a través de la Ley 2/1989, de 18 de julio, por la que se aprueba el Inventario de Espacios Naturales Protegidos de Andalucía, y se establecen medidas adicionales para su protección. Esta figura equivaldría a la categoría V de la UICN (Conservación de de paisajes terrestres y marinos y recreación). En relación con esta analogía, y como se deduce de esta cronología, su reconocimiento responde a criterios en los que predomina la conservación sobre el desarrollo sostenible, un concepto que aún estaba por aparecer. De hecho, la incorporación de los Planes de Uso Público a dicha normativa es muy reciente ${ }^{2}$, primando objetivos de conservación.

En concreto, es la amenaza de dos especies, pinsapo (Abies pinsapo bois) y, en menor medida, el quejigo (Quercus faginea ssp. Faginea) la que se esgrime como causa principal de la declaración. Así, el pinsapo tiene carácter relictual respecto de la flora terciaria y su localización exclusiva en distintos puntos de

2 El Programa de Uso Público del parque natural Sierra de las Nieves se aprueba un año después de la aprobación de los nuevos PORN y PRUG por "Resolución del Director General de la Red de Espacios Naturales Protegidos y servicios ambientales de 4 de febrero de 2004 por la que se aprueba el Programa de Uso Público del parque natural Sierra de las Nieves". Como este enunciado indica, la entonces reciente creación de la Red de Espacios Naturales Protegidos de la comunidad autónoma de Andalucía (de ahora en adelante RENPA) en 2003 está en la base de sus objetivos y contenido. En los "Antecedentes" de este PUP encontramos un reconocimiento de la incorporación a posteriori del uso público en la gestión de los espacios naturales protegidos declarados en la ley 2/1989, proponiendo el documento "Gestión del uso público en la RENPA. Estrategia de Acción" (2003) como base metodológica seguida en su redacción. En este mismo epígrafe se reconoce al PUP como desarrollo del PRUG ya analizado. 
la Serranía de Ronda (Grazalema en Cádiz, $S^{a}$ Bermeja y $S^{a}$ de las Nieves en Málaga) y en la cordillera de Yebala, al norte de Marruecos, son los factores que aportan su valor desde la perspectiva de la biodiversidad, mientras que los procesos de desforestación, continuos desde el s. XV, habían conducido a una clara situación de riesgo de desaparición, del que era consciente la administración forestal del Estado desde décadas anteriores. Aunque más adelante (1995) su espacio (ampliado) es declarado como Reserva de la Biosfera, al carecer esta figura en España de normas de zonificación y gestión específicas, es la normativa de parque natural la que determina la configuración territorial del parque, que responde al objetivo de envolver y recoger la discontinua presencia de pinsapos sobre las alineaciones más orientales de la Serranía de Ronda, donde se acantonaron en sus cañadas más umbrías, cuya altitud y orientación les proporcionaban la humedad y frescor que permitieron su supervivencia. Siguiendo los cánones de la zonificación proteccionista, son estas formaciones las que configuran el núcleo de mayor protección de la zonificación prevista por el Plan de Ordenación de Recursos Naturales (PORN de ahora en adelante) aunque en ésta se reconoció con la máxima protección otras áreas aisladas dotadas de otras formaciones vegetales igualmente valiosas, estableciéndose un área periférica a la localización del pinsapar.

Esta delimitación carece de continuidad histórica. En la organización política-territorial correspondiente a la fase de economía orgánica (después de la repoblación cristiana, a finales del s. XVI) (GÓMEZ, 1989), la $S^{\text {a }}$ de las Nieves era utilizada, por su envergadura, como hito natural en torno al cual se abrían los concejos de Marbella, al Sur, Ronda, al Norte, y Málaga, al Este, siguiendo, aproximadamente, la divisoria de aguas. Desde esta perspectiva histórica que recoge funciones muy distintas de las actuales, se entiende que el poblamiento se ubique fuera de lo que hoy es el parque natural. Con la excepción de Ronda, que responde al modelo de ciudades medias localizadas en el Surco Intrabético desde la Edad Antigua, el resto de los municipios recoge un hábitat consolidado desde la Baja Edad Media y que buscaba el emplazamiento en torno a las surgencias, muchas veces asociadas a formaciones travertínicas, resultantes de la superposición de materiales permeables (mármoles, calizas en la zona de cumbre) sobre impermeables (esquistos, gneises, en los piedemontes) ligados a las Unidades Internas de las Béticas que configuran los tramos meridional y oriental de estas sierras. Otro factor de localización es la exposición, buscando la solana, Oriente y Occidente y eludiendo las umbrías. De esta forma, el ager bajo propiedad privada minifundista se situaba en los piedemontes, yuxtaponiendo secanos (arboricultura: almendros, higueras, vides, olivos) y regadíos (hortofrutícolas en los travertinos y en las terrazas sobre valles encajados) y el saltus, bajo propiedad colectiva pública, en las zonas más altas, proporcionaba aprovechamientos silvopastoriles a cada uno de estos concejos ligados a desplazamientos temporales de la mano de obra correspondiente, fundamentalmente 
pastores, carboneros y neveros. Así mismo, su posición central le convertía en paso obligado de los caminos que conectaban los valles orientales con sus productos hortofrutícolas y los núcleos costeros (pesca) con las más continentalizadas tierras de Ronda, Norte de Cádiz y Sur de Sevilla. En síntesis, lo que hoy es el parque natural era un territorio forestal, despoblado y de paso y puesto en valor por poblaciones muy distantes entre sí y que tenían en la agricultura de vertiente su principal actividad.

El hábitat de estos municipios es actualmente muy homogéneo (entre 1.500 y 3.500 habitantes) tras haber experimentado las fases de crecimiento demográfico que revelan el paso al modelo del capitalismo industrial, con un fuerte éxodo rural entre 1958 y 1975, envejecimiento y decrecimiento vegetativo en la década de los ochenta y primeros noventa e inmigración de población madura, tanto retornados como extranjeros para residencia estacional, desde finales de los Noventa.

La proximidad relativa a la Costa del Sol (un centro turístico de relevancia mundial y que reúne en torno al millón de habitantes permanentes) y la debilidad de los ingresos proporcionados por las actividades agrarias (RUBIO, GÓMEZ y BLANCO, 2010) ha condicionado su sistema productivo. Comenzando por los primeros, el predominio del minifundismo unido a los bajos precios e origen imposibilita que la explotación agraria sea la única fuente de ingresos de los habitantes. De esta forma, los ingresos de las familias son una suma de los proporcionados por la agricultura (sólo el 18\% de los ocupados se declaran ocupados en este sector según el Censo de Población del 2001, el único disponible), predominando el sector de la construcción (el 28\%) con un 9,7\% para la industria, 9,6\% para la hostelería y sólo un $10 \%$ para el comercio. Pero el auténtico problema de estos municipios, como el de la mayor parte de los municipios rurales andaluces, sean o no de montaña, es el paro. En la Fig. 1se puede comprobar como el paro presenta su nivel más bajo en la etapa del denominado "boom inmobiliario" para dispararse al entrar en la fase de crisis económica actual, demostrando así la mayor vinculación de la economía de estos municipios a la actividad costera que a la local.

Si retomamos los factores expuestos, queda por desarrollar como los objetivos de la gestión del parque natural son obsoletos respecto a los nuevos planteamientos de la UICN. En el cuadro 2 se puede observar cómo predominan absolutamente los objetivos de conservación y educación ambiental orientada al uso público por los visitantes en detrimento de los orientados al desarrollo sostenible. Pero hay otro elemento de este sistema de gestión que pone claramente de manifiesto la débil interacción entre éste y los actores locales: la composición de las juntas rectoras de los parques naturales fijada por la legislación andaluza (ver cuadro 3) limita al 10\% de sus componentes la presencia de los representantes de los ayuntamientos, correspondiendo todos los demás a representantes de organismos de la administración y de asociaciones ciuda- 
danas pero no necesariamente vinculadas a los municipios del parque natural. Por ejemplo, está prevista la presencia de un representante de asociaciones de empresarios y otro de los sindicatos, pero ni uno ni otro deben residir en dichos municipios. Por tanto, estamos lejos del fortalecimiento de la acción de los agentes locales en la gestión del parque natural que proponen los nuevos planteamientos de la UICN (DUDLEY, 2008).

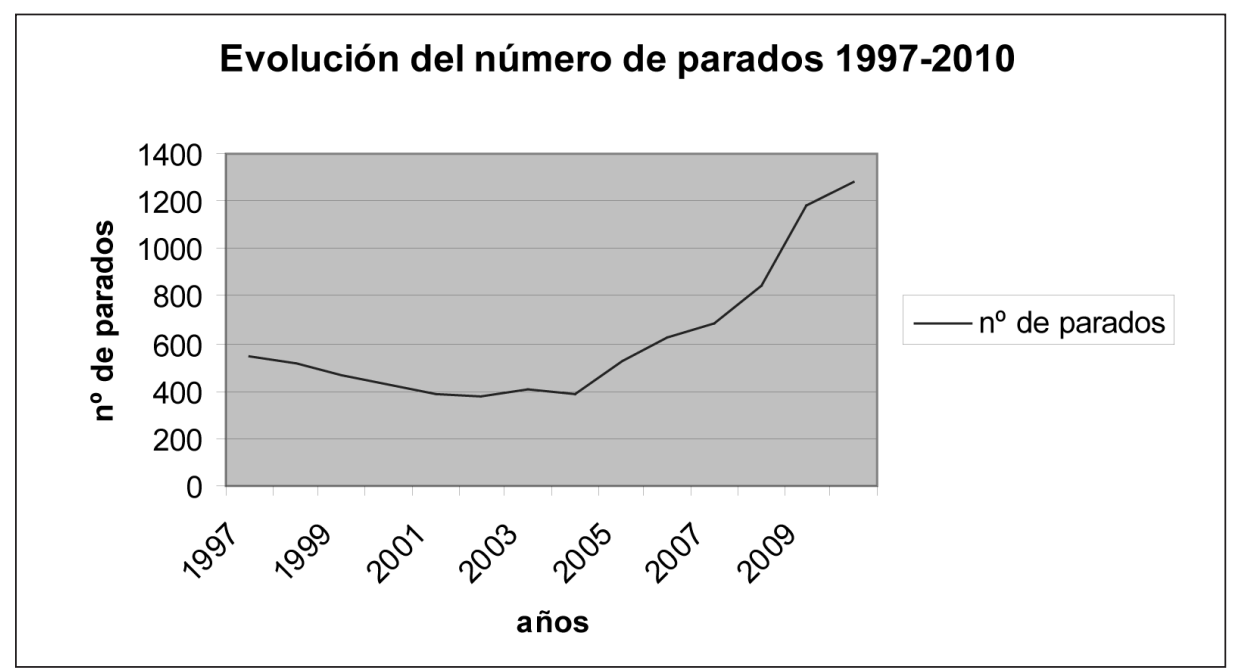

Figura 1. Evolución del número de parados 1997-2010.

Fuente: 1997-2004 INEM; 2005-2010 Consejería de Empleo - Servicio Andaluz de Empleo acceso: SIMA.

Por último, los resultados de la encuesta enunciada en el epígrafe 1 pusieron de manifiesto dos hechos que cuestionan la orientación de la gestión y, como adelantábamos, la capacidad que esta "visibilidad" del espacio que supone la declaración como parque tiene de activar el tejido productivo de las zonas rurales en que se inserta. En el cuadro 4 hemos recogido los resultados de la encuesta respecto a cuál es la motivación del usuario de los senderos de uso público. Como se puede comprobar, el número de usuarios cuya motivación es el interés por el conocimiento de los valores naturales o culturales del parque es muy inferior al de aquellos que, simplemente, quieren estar en contacto con la naturaleza, hacer deporte al aire libre o estar con los amigos. Por tanto, realmente, la mayor parte de la población urbana que visita los parques naturales no lo hace con una intención de educación ambiental.

En el cuadro 5 hemos recogido los resultados de la encuesta respecto a la incidencia del uso público (senderismo) en el tejido productivo local. Cómo se puede comprobar, entre el 75 y el 805 de los senderistas encuestados no ha recurrido a ninguno de los servicios privados de éste. No se ha alojado, no 
Cuadro 2. Comparación entre los objetivos de gestión iniciales de la Categoría V de la UICN y los objetivos del Plan de Ordenación de Recursos Naturales del parque natural de Sierra de las Nieves

\begin{tabular}{|c|c|}
\hline $\begin{array}{c}\text { Objetivos de gestión iniciales de la } \\
\text { Categoría V de la UICN }\end{array}$ & Objetivos PORN $S^{\text {a }}$ de las Nieves \\
\hline $\begin{array}{l}\text { 3.-Conservar la diversidad del paisaje y } \\
\text { el hábitat, y de las especies y ecosistemas } \\
\text { asociados } \\
\text { 4.-Excluir cuando sea necesario, y por lo } \\
\text { tanto prevenir, las modalidades de utiliza- } \\
\text { ción de tierras y las actividades de carác- } \\
\text { ter y/o magnitud inadecuados }\end{array}$ & $\begin{array}{l}\text { 1.-La continuidad de los pinsapares por } \\
\text { su valor ecológico y como formación em- } \\
\text { blemática del PN } \\
\text { 2.-La conservación y regeneración de la } \\
\text { masa forestal como complemento pro- } \\
\text { tector del suelo contra la erosión y como } \\
\text { mecanismo que contribuye a frenar el } \\
\text { cambio climático. } \\
\text { 4.-La conservación de la geodiversidad y } \\
\text { la diversidad ecológica y de especies, con } \\
\text { especial atención a los hábitats y especies } \\
\text { catalogadas de interés comunitario. } \\
\text { 5.-Mantener en buen estado la calidad de } \\
\text { los recursos hídricos }\end{array}$ \\
\hline $\begin{array}{l}\text { 1.-Preservar la interacción armoniosa en- } \\
\text { tre la naturaleza y la cultura, a través de la } \\
\text { protección de paisajes terrestres y/o ma- } \\
\text { rinos y el mantenimiento de las prácticas } \\
\text { tradicionales de utilización de tierras, los } \\
\text { métodos de construcción y las manifesta- } \\
\text { ciones sociales y culturales } \\
\text { 2.-Promover estilos de vida y actividades } \\
\text { económicas que estén en armonía con la } \\
\text { naturaleza y la preservación de la trama } \\
\text { social y cultural de las comunidades con- } \\
\text { cernientes } \\
\text { 6.-Alentar las actividades científicas y } \\
\text { educativas que contribuyan al bienestar a } \\
\text { largo plazo de las poblaciones residentes } \\
\text { y a estimular el apoyo público en favor de } \\
\text { la protección ambiental de dichas áreas } \\
\text { 8.-Aportar beneficios a las comunidades } \\
\text { locales, y contribuir a su bienestar, a tra- } \\
\text { vés del suministro de productos naturales } \\
\text { (como los derivados de los bosques y la } \\
\text { pesca) y la prestación de servicios (como } \\
\text { abastecimiento de agua potable o genera- } \\
\text { ción de ingresos a partir de formas soste- } \\
\text { nibles de turismo) }\end{array}$ & $\begin{array}{l}\text { 3.-Compatibilizar los usos y actividades } \\
\text { con la conservación de los recursos natu- } \\
\text { rales } \\
\text { 6.-Facilitar las condiciones socioeco- } \\
\text { nómicas que eviten el desarraigo de las } \\
\text { comunidades rurales y favorezcan su pro- } \\
\text { greso, promoviendo un uso económico } \\
\text { y social del territorio compatible con la } \\
\text { conservación de los recursos naturales } \\
\text { 8.-Poner en valor el patrimonio cultural } \\
\text { del PN desde una óptica integrada, como } \\
\text { recurso potencial en las políticas de desa- } \\
\text { rrollo sostenible, promoviendo su utiliza- } \\
\text { ción racional como instrumento de desa- } \\
\text { rrollo cultural y económico siempre que } \\
\text { no suponga un menos cabo o deterioro de } \\
\text { los valores naturales. } \\
\text { 10.-El adecuado desarrollo de las edifica- } \\
\text { ciones e infraestructuras que deban ubi- } \\
\text { carse en el espacio, protegiendo la cali- } \\
\text { dad visual e identidad paisajística de este } \\
\text { espacio. }\end{array}$ \\
\hline
\end{tabular}




\begin{tabular}{|l|l|}
\hline \multicolumn{1}{|c|}{$\begin{array}{c}\text { Objetivos de gestión iniciales de la } \\
\text { Categoría V de la UICN }\end{array}$} & \multicolumn{1}{c|}{ Objetivos PORN S de las Nieves } \\
\hline $\begin{array}{l}\text { 5.-Ofrecer oportunidades de esparcimien- } \\
\text { to público a través de formas de recrea- } \\
\text { ción y turismo que estén en consonancia, } \\
\text { por su carácter y magnitud, con las cali- } \\
\text { dades esenciales de estas áreas }\end{array}$ & $\begin{array}{l}\text { 7. Fomentar el papel del PN en el desa- } \\
\text { rollo de las actividades de uso público } \\
\text { de forma compatible con la conservación } \\
\text { de locursos naturales, permitiendo el } \\
\text { uso y disfrute de los ciudadanos así como } \\
\text { el acercamiento a sus valores naturales y } \\
\text { culturales. } \\
\text { 9. Integrar los valores del patrimonio na- } \\
\text { tural y cultural en el desarrollo de progra- } \\
\text { mas educativos que promuevan una con- } \\
\text { ciencia social favorable a la conservación } \\
\text { de los mismos }\end{array}$ \\
\hline
\end{tabular}

Fuente.: UICN: http://intranet.catie.ac.cr/intranet/posgrado/Manejo\%20Areas\%20Protegidas/ Documentos/_CATEGORIAS\%20DE\%20MANEJO/_GUIA\%20CATEGORIAS\%20DE\%20 MANEJO/Categorias\%20de\%20Manejo\%20UICN\%202004.pdf

PORN Sierra de las Nieves http://www.juntadeandalucia.es/medioambiente/web/Bloques_Tematicos/Patrimonio_Natural._Uso_Y_Gestion/Espacios_Protegidos/PORN/PORN_PRUG_Sierra_Nieves/anexo3snieves.pdf

Cuadro 3. Composición de las juntas rectoras de los parques naturales de Andalucía

\begin{tabular}{|l|c|}
\hline \multicolumn{1}{|c|}{ Representantes } & $\%$ \\
\hline Representantes del gobierno autonómico & 22 \\
\hline Representantes del gobierno provincial & 2 \\
\hline Representantes del gobierno local & 14 \\
\hline Representantes del gobierno nacional & 2 \\
\hline Organizaciones socioeconómicas y empresariales & 20 \\
\hline $\begin{array}{l}\text { Tres representantes a propuesta de la Federación andaluza de Deportes, } \\
\text { preferentemente de las legalmente registradas en los municipios correspon- } \\
\text { dientes al P.N. }\end{array}$ & 7 \\
\hline Organizaciones ciudadanas & 11 \\
\hline Un representante por cada grupo parlamentario del Parlamento Andaluz & 9 \\
\hline Instituciones científicas & 7 \\
\hline Miembros de reconocido prestigio & 7 \\
\hline & 100 \\
\hline
\end{tabular}

Fuente.: Elaborado a partir de las composiciones previstas por el Decreto 239/1997 de 15 de octubre, por el que se regula la constitución, composición y funciones de las Juntas Rectoras de los Parques Naturales. 
Cuadro 4. Motivación del usuario de los senderos de uso público

\begin{tabular}{|l|c|c|}
\hline \multicolumn{1}{|c|}{ RESPUESTAS } & $\mathrm{N}^{\mathrm{o}}$ & $\%$ \\
\hline Pasar un día de campo & 128 & 37,10 \\
\hline Hacer deportes de naturaleza & 124 & 35,94 \\
\hline Hacer deportes de naturaleza y otras & 6 & 1,74 \\
\hline Pasar un día de campo y hacer deporte en la naturaleza & 26 & 7,54 \\
\hline Ver alguna cosa específica & 16 & 4,64 \\
\hline Otras & 32 & 9,28 \\
\hline $\begin{array}{l}\text { Pasar un día de campo, ver alguna cosa específica y hacer deporte } \\
\text { en la naturaleza }\end{array}$ & 5 & 1,45 \\
\hline $\begin{array}{l}\text { Pasar un día de campo, ver alguna cosa específica hacer deporte en } \\
\text { la naturaleza y otras }\end{array}$ & 2 & 0,58 \\
\hline Pasar un día de campo y ver alguna cosa específica & 2 & 0,58 \\
\hline Pasar un día de campo y otras & 3 & 0,87 \\
\hline Ver alguna cosa específica y hacer deporte en la naturaleza & 1 & 0,29 \\
\hline & 345 & 100 \\
\hline
\end{tabular}

Fuente: Encuesta Proyectos referencia SEJ-2007-67690 y P07_HUM_03049

Cuadro 5. Utilización de servicios privados en los municipios del área de influencia del parque natural por los usuarios de los senderos de uso público

\begin{tabular}{|l|c|c|c|c|c|c|}
\hline \multirow{2}{*}{ Preguntas } & \multicolumn{5}{|c|}{ Respuestas } \\
\cline { 2 - 8 } & \multicolumn{2}{|c|}{ Sí } & \multicolumn{2}{c|}{ No } & \multicolumn{2}{c|}{ Total } \\
\hline & Total & $\%$ & Total & $\%$ & Total & $\%$ \\
\hline $\begin{array}{l}\text { ¿Se ha alojado en algún } \\
\text { municipio de la zona? }\end{array}$ & 38 & 11 & 307 & 89 & 345 & 100 \\
\hline $\begin{array}{l}\text { ¿Ha consumido comida traída de } \\
\text { casa? }\end{array}$ & 295 & 85,5 & 50 & 14,5 & 345 & 100 \\
\hline $\begin{array}{l}\text { ¿Ha realizado o piensa realizar } \\
\text { compras en establecimientos de } \\
\text { la zona? }\end{array}$ & 270 & 22 & 75 & 78 & 345 & 100 \\
\hline
\end{tabular}

Fuente: elaboración propia. 
ha ido a restaurantes ni ha comprado en los comercios locales. Sin embargo, otros estudios realizados en el marco del proyecto y centrados en entrevistas y estudios económicos de estos servicios privados están revelando que sí cuentan con una clientela que, en muchas ocasiones, no recurre al uso de estos servicios públicos. Por tanto, queda claro que la respuesta a la pregunta de Benós et al. Es que la interacción entre protección y componentes de la estructura socioeconómica es muy baja y no funciona bajo los principios del desarrollo local.

\subsection{Discusión: Recuperación de la interrelación ager saltus, del liderazgo de los agentes locales, de la rentabilidad de los sistemas agrarios mediante el cierre del ciclo agroalimentario}

Desde nuestro punto de vista, esta situación de las áreas de montaña mediterránea puede mejorar si se aplica la "Estrategia Europa 2020" y como consecuencia de la ampliación de la superficie protegida en áreas de montaña derivada de la aplicación tanto de la Red Natura 2000 como de la Ley 42/2007 de 13 de diciembre del Patrimonio natural y la biodiversidad.

En lo que se refiere a "Europa 2020", sus planteamientos sobre crecimiento económico proporcionan, al menos en teoría, nuevas bases. En primer lugar, en la medida en que supone la consideración de la sostenibilidad ("una Europa que utilice eficazmente sus recursos") como un objetivo común a todas las actividades y territorios. Ello debería implicar la sustitución de la función territorial que actualmente tiene asignada de forma más genérica, la conservación de espacios naturales, por una inserción más activa y compatible con el mantenimiento de estos valores naturales, tanto más cuanto muchos de estos espacios no se corresponden con espacios "salvajes" sino con espacios antropizados por sistemas de uso poco intensivos y ligados a la economía orgánica. En segundo lugar, y ligado con lo anterior, por la función que asigna a la innovación a través de dos dimensiones: la vinculación de ésta a los procesos productivos y la recualificación de los recursos humanos. El uso de las TIGs, la vinculación entre conocimiento reglado académico y conocimiento informal de los habitantes montañeses, es un procedimiento fundamental para alcanzar esa mejor inserción productiva al facilitar el conocimiento de los ciclos productivos naturales a escalas detalladas y, con ello, llegar a propuestas concretas de formas de producción de calidad compatibles con la estabilidad ambiental y la diversidad de los paisajes. A su vez, ello redundaría en una oportunidad de venta de los productos a los visitantes, clave para la articulación entre uso turístico y sistemas agroambientales. Por último, el concepto de "Economic governance" implica que los agentes locales deben tener una nueva y reforzada función en la gestión de los parques naturales.

En lo que respecta a la normativa ambiental aludida, estas oportunidades derivadas de las propuestas socioeconómicas de "Europa 2020" se extenderían 
a una superficie muy significativa debido a que la relación entre montaña mediterránea y espacios protegidos va a ampliarse debido a dos hechos. Uno es que buena parte del incremento del espacio protegido inducido por la aplicación de los LIC se sitúa en aquella. Otro es la especial consideración que las áreas de montaña (sean o no mediterráneas) reciben en la citada Ley 42/2007. Ambos hechos implican, a medio y largo plazo, la debilidad de los límites entre los diferentes instrumentos de conservación y sus correspondientes sistemas de gestión, y, por tanto, la búsqueda de alternativas a éstos que pueden seguir los principios de "Europa 2020" antes expuestos.

En consecuencia, el sistema de gestión actual de los parques naturales españoles debe modificarse para materializar las posibilidades de dinamización económica que la vinculación de los conceptos de visibilidad y demanda de Rural Goods and Services suponen para las áreas de montaña mediterránea.

\section{Conclusiones}

1.-La perspectiva teórica del desarrollo local proporciona una interpretación adecuada de las razones por las que las posibilidades de dinamización económica que supone la localización de la mayor parte de los parques naturales sobre zonas de montaña mediterránea no se están materializando. Desde esta perspectiva, estas razones serían dos factores interrelacionados: la debilidad cuantitativa y cualitativa del capital humano y el sistema de gestión en el que la presencia y participación del «capital relacional » de los municipios está muy limitada tanto por la propia configuración de su representación (las juntas rectoras) como por las escasas competencias a ellas reservadas.

2.-La prioridad del objetivo de educación ambiental en esta gestión no coincide con la motivación de los visitantes usuarios de los equipamientos de uso público, más orientada hacia el concepto de Rural Good and Services. Este hecho puede ser interpretado como nuevas oportunidades para las áreas de montaña con calidad ambiental, estén o no protegidas bajo criterios de biodiversidad.

3.-Sin embargo, estas oportunidades no pueden residir únicamente en las actividades turísticas, puesto que los resultados ponen de manifiesto un débil gasto de los visitantes en las mismas.

4.-En este sentido, la combinación de las formulaciones estratégicas de "Europa 2020" con la extensión de las zonas protegidas en áreas de montaña, deben ser la base para la aplicación de nuevas formas de gestión de éstas que permitan recuperar la interacción de ager y saltus en sistemas productivos que combinen calidad ambiental y calidad de la producción (trazabilidad) basándose en el uso de nuevas tecnologías y en una participación real de los actores locales. 
BIBLIOGRAFÍA

BENOS, R. CAZENAVE, A. Y MILIAN, J. (2007): "Pirineos-Monte Perdido Patrimonio mundial: un espacio de montaña frente a su protección y gestión", en III Coloquio Hispano-Francés de Geografía Rural / IIIe Colloque Franco-Espagnol de Géographie Rurale. Ed. CD-Rom AGE, UNIA sede Antonio Machado, CNFG. pp. 47-63, p. 47.

DEMATTEIS, G. Y GOVERNA, F. (2005): Territorio y territorialidad en el desarrollo local. La contribución del modelo SLOT in Boletín de la Asociación de Geógrafos Españoles, nº 39, p. 31-58.

DUDLEY, N. (Editor) (2008). Guidelines for Applying Protected Area Management Categories. Gland, Switzerland. Available at: http://data.iucn.org/ dbtw-wpd/edocs/paps-016.pdf consultado en noviembre de 2012.

ESPARCIA, J. Y BUCIEGA, A. (2005): New rural-urban relationships in Europe: A comparative Analysis. Experiences from The Netherlands, Spain, Hungary, Finland and France. Ed. Universitat de Valéncia e Instituto Interuniversitario de Desarrollo Local.

Estrategia Europa 2020 http://ec.europa.eu/commission_2010-2014/president/ news/documents/pdf/20100303_1_es.pdf

GOMEZ, M.L. (1989): La montaña malagueña. Estudio ambiental y evolución de su paisaje. Ed. Serv. Public. Diput. Prov. de Málaga, Málaga, pp.412.

GOMEZ, M.L. (2008): "La función de la montaña en los territorios ibéricos: validez de la perspectiva unificada de la geografía" Conferencia de clausura CONGRESO: XI Coloquio Ibérico de Geografía Alcalá de Henares - Pastrana, del 1 al 4 de octubre de 2008. PUBLICACIÓN: CD-Rom La perspectiva geográfica ante los retos de la sociedad y el medio ambiente en el contexto ibérico, Ponencias del XI Coloquio Ibérico de Geografía, Serv. De Publicaciones de la Universidad de Alcalá. Madrid, 2009, pp. 149-179. ISBN978-84-8138-792-6. LA FUNCIÓN DE LA MONTAÑA EN LOS TERRITORIOS IBÉRICOS: VALIDEZ DE ...www.geogra.uah.es/inicio/ web_11.../Clausura_MLuisa_Gomez.pdf

IUCN (1994): Commission on National Parks and Protected Areas, Guidelines for protected areas management URL: http://books.google.es/books?id=4 gi3vtvRJ9EC\&dq=UICN+cATEGOR\%C3\%8DAS,+OBJETIVOS+Y+CRI TERIOS+PARA+LAS+\%C3\%81REAS+PROTEGIDAS+1978\&hl=es\&so urce $=$ gbs_navlinks_s. Consulta noviembre de 2012.

OCAÑA, C.; GÓMEZ, M L L.; ARREBOLA, J.A. BLANCO, R.; GALACHO, F.B.; LARRUBIA, R.; LUQUE, A.; MÉRIDA, M.; NAVARRO, S.; RUBIO, L.M.; VÍAS, J. (2013): "Desarrollo metodológico sobre la evaluación de la capacidad de uso recreativo de espacios protegidos", Nimbus, 12-13, en prensa. 
PULIDO FERNÁNDEZ, J. I. (2008): “Gestión turística y desarrollo económico en los parques naturales andaluces. Una propuesta de revisión desde el análisis del posicionamiento de sus actuales gestores", Revista de Estudios Regionales, $\mathrm{n}^{\circ}$ 81, p. 171-203.

RUBIO, L.M., GÓMEZ, M M L. Y BLANCO, R. (2010): "Sostenibilidad y orientación de las explotaciones en terrazas de cultivo en regadío" en Papeles de Geografía de la Universidad de Murcia, n $^{\circ}$ 51-52, p. 257-267.

WRIGLEY, E.A. (1988): Continuity, chance and change. The character of the industrial revolution in England, Cambridge University Press, United Kingdom.

ZOIDO, F. y CARAVACA, I. (eds.) (2005): Andalucía. Segundo informe de desarrollo territorial. Ed. Fundación Sevillana Endesa, Univ. De Sevilla, Cons. De Innovación, Ciencia y Empresa de la Junta de Andalucía y Secretariado de Publicaciones de la Univ. De Sevilla. p. 413.

\section{Legislación y normativa consultada}

Ley 4/1989 de 27 de marzo de Conservación de los Espacios Naturales y de la Flora y Fauna Silvestres

Ley 2/1989, de 18 de julio, por la que se aprueba el Inventario de Espacios Naturales Protegidos de Andalucía y se establecen medidas adicionales para su protección.

Ley 6/1996 de 18 de julio relativa a la modificación del artículo 20 de la Ley 2/1989 de 18 de julio por la que se aprueba el inventario de espacios naturales protegidos de Andalucía, desarrollada por el Decreto 239/1997 de 15 de octubre, por el que se regula la constitución, composición y funciones de las Juntas Rectoras de los Parques Naturales

Ley 42/2007 de 13 de diciembre del Patrimonio natural y la biodiversidad Decreto 15/2011, de 1 de febrero, por el que se establece el régimen general de planificación de los usos y actividades en los parques naturales y se aprueban medidas de agilización de procedimientos administrativos.

http://www.juntadeandalucia.es/medioambiente/web/Bloques_Tematicos/Patrimonio_Natural._Uso_Y_Gestion/Espacios_Protegidos/PORN/PORN_ PRUG_Sierra_Nieves/anexo3snieves.pdf consulta 11 de octubre de 2011 http://intranet.catie.ac.cr/intranet/posgrado/Manejo\%20Areas\%20Protegidas/ Documentos/_CATEGORIAS\%20DE\%20MANEJO/_GUIA\%20CATEGORIAS\%20DE\%20MANEJO/Categorias\%20de\%20Manejo $\% 20$ UICN\%202004.pdf consulta 15 de mayo de 2013 